\title{
On the Competition among Three Modes of Innovation: Incubative Innovation, Acquisitive Innovation and Shanzhai
}

\author{
Yinxin Lu \\ Management School, Jinan University, Guangzhou, China \\ Email: lu yinxin@hotmail.com
}

Received 10 March 2015; accepted 6 April 2015; published 9 April 2015

Copyright ( 2015 by author and Scientific Research Publishing Inc.

This work is licensed under the Creative Commons Attribution International License (CC BY). http://creativecommons.org/licenses/by/4.0/

c) (i) Open Access

\begin{abstract}
In this article, we investigate how Shanzhai phenomenon affects the competition among firms adopted different innovation mode, namely incubative innovation, acquisitive innovation and Shanzhai. Using the framework of vertical product differentiation model, we first analyze the duopoly game between incubative innovation and acquisitive innovation, then introduce Shanzhai innovation to the model, and compare the equilibrium result between the two games. We show that the rise of Shanzhai innovation has the opposite effect on the innovation incentive of incubative innovator and acquisitive innovator. The rise of Shanzhai innovation also decreases the prices and profits of incubative innovator and acquisitive innovator, decreases producer surplus and social welfare, while increases consumer surplus. Moreover, if the efficiency parameter of Shanzhai innovation is sufficiently small, firms adopted Shanzhai innovation might earn higher profits than the ones adopted acquisitive innovation and incubative innovation.
\end{abstract}

\section{Keywords}

Innovation Mode, Incubative Innovation, Acquisitive Innovation, Shanzhai

\section{Introduction}

It is widely accepted that firms need to develop new product innovations in order to remain competitive in today's business markets [1]. Product innovation can be defined as the market introduction of a product that is new to the firm or its market [2]. In China, firms follow three different patterns in developing or acquiring innovation: incubative innovation, acquisitive innovation or Shanzhai. Incubative innovation reflects the firm's disposition to develop its own innovations (e.g., R and D) [3]. Since incubative innovation is costly, risky and path-depen- 
dent, quite a few firms choose to simply acquire foreign technology created in developed countries or other external sources. Thus, acquisitive innovation reflects a firm's disposition to acquire innovations that have been developed by other firms through purchase, licensing, acquisition, merger or joint venture [3]. Through acquisitive innovation, a firm may gain quick access to the innovations it needs. In most cases, however, firms that adopt acquisitive innovation could not acquire the most advanced technology due to the private interest of the origin innovator. Since 2003, Shanzhai phenomenon spread across China, and Shanzhai mode emerged as a new innovation mode. The term Shanzhai, indicating small-scale factories or small family-run workshops, was introduced from Hong Kong to mainland China. Shanzhai, known for low-price and low-quality, has been indirectly described as imitators, knock-offs, and copycats due to its phenomenal imitation activities [4]. In fact, Shanzhai products have gone far beyond piracy and counterfeits and Shanzhai is regarded as imitative innovation [4], though it has very limited innovation output such as IPRs. In this paper, Shanzhai refers to imitative innovation, reflecting a firm's disposition to copy innovations first introduced by its rivals or firms in other industries [3]. Due to the low-cost and low-threshold of Shanzhai, firms adopted this innovation mode have already become a non-negligible force in the market. In this paper, we would like to investigate how Shanzhai influences the other two innovation modes and thus imposes impact on the market equilibrium.

The existing studies on innovation mode, such as [5]-[7], focus on one particular innovation mode. Some other studies have considered two or more innovation modes simultaneously [3] [4]. However, a paucity of research has studied the competition between different innovation modes. Since quite a few studies have referred to the vertical product differentiation framework in analyzing product innovation [8]-[10], here we adopt the same framework to analyze the competition between different innovation modes.

The paper is organized as follows. Section 2 describes the duopoly game before Shanzhai phenomenon emerged. Section 3 introduces Shanzhai manufacturer into the model to depict the emergence of Shanzhai phenomenon. Section 4 compares the result of the model before and after Shanzhai phenomenon emerged. Section 5 concludes the paper.

\section{Duopoly Game before Shanzhai Phenomenon Emerged}

We consider a vertical differentiated model following the structure of [10]. Consumers either buy one unit of one good, or buy nothing. Before Shanzhai phenomenon emerged, there are two firms in the market, firm $n$ and firm $a$, indicating firms adopted incubative innovation and acquisitive innovation (hereafter referred as incubative innovator and acquisitive innovator respectively). Each firm produces one variant of product and decides on its price. As mentioned above, the technology acquired from external would be less advanced than that generated internally. Hence, we assume that, the quality of the product developed by firm $n$ is higher than firm $a$, namely $S_{n}>S_{a}$.

We assume that the two firms are selling products to a population of consumers differing in their taste for quality. We denote individual consumer taste as $\theta$, which is uniformly distributed over the interval $[0, \bar{\theta}]$, with density 1 . A consumer, identified by $\theta$, enjoys utility $u(\theta)=\theta S-P$ when consuming a product of quality Sat price $P$. His utility is zero if he refrains from buying. Thus demand addressed to firm $i$ is defined by the set of consumers who maximize utility when buying product $i$, rather than product $j$ or refraining from buying. The consumer indifferent between consuming the product developed by incubative innovator and not buying at all has a utility given by $\theta_{1} S_{a}-P_{a}=0$, so that $\theta_{1}=P_{a} / S_{a}$. The consumer indifferent between consuming the product developed by incubative innovatoror the product developed by acquisitive innovator has a taste parameter satisfying $\theta_{2} S_{a}-P_{a}=\theta_{2} S_{n}-P_{n}$, with $\theta_{2}=\left(P_{n}-P_{a}\right) /\left(S_{n}-S_{a}\right)$. Hence consumers for whom $\theta \in\left[\theta_{2}, \bar{\theta}\right]$ choose the product offered by incubative innovator, while those described by $\theta \in\left[\theta_{1}, \theta_{2}\right]$ choose the product offered by acquisitive innovator. The one described by $\theta \in\left[0, \theta_{1}\right]$ do not consume at all. Denote the variables in this case with a superscript $D$, hence the market shares for the two varieties are, respectively,

$$
\begin{aligned}
& d_{a}^{D}=\frac{P_{n}-P_{a}}{S_{n}-S_{a}}-\frac{P_{a}}{S_{a}} \\
& d_{n}^{D}=\bar{\theta}-\frac{P_{n}-P_{a}}{S_{n}-S_{a}}
\end{aligned}
$$

In the duopoly, each firm $i$ offer one quality $S_{i}$ and faced a R\&D cost $C\left(S_{i}\right)$ that enables the provision of 
the quality. The quality cost is a standard quadratic function $C\left(S_{i}\right)=\gamma \cdot S_{i}^{2} / 2, \gamma>0$, where $\gamma$ is R\&D cost parameter, and higher values of $\gamma$ indicates less efficient at $\mathrm{R} \& \mathrm{D}$. Thus the profits are given by:

$$
\begin{aligned}
& \Pi_{n}^{D}=\left(\bar{\theta}-\frac{P_{n}-P_{a}}{S_{n}-S_{a}}\right) P_{n}-\frac{\gamma \cdot S_{n}^{2}}{2} \\
& \Pi_{a}^{D}=\left(\frac{P_{n}-P_{a}}{S_{n}-S_{a}}-\frac{P_{a}}{S_{a}}\right) P_{a}-\frac{\gamma \cdot S_{a}^{2}}{2}
\end{aligned}
$$

The competition between firms takes place in a two-stage game. In the first stage, they choose whether to enter the market, and decide on the quality $S_{i}$ to develop if enter. We assume that a firm will not enter the market unless it earns a positive profit. In the second stage, firms choose prices $P_{i}$.

The game is solved by backward induction in order to provide the subgame perfect equilibrium. In the second stage, firms choose their price taking as fixed qualities $S_{n}$ and $S_{a}$. By differentiating Equations (3) and (4), one obtains the following first-order conditions:

$$
\begin{gathered}
\frac{\partial \Pi_{n}^{D}}{\partial P_{n}}=\frac{\bar{\theta}\left(S_{n}-S_{a}\right)-2 P_{n}+P_{a}}{S_{n}-S_{a}}=0 \\
\frac{\partial \Pi_{a}^{D}}{\partial P_{a}}=\frac{P_{n} S_{a}-2 P_{a} S_{n}}{S_{a}\left(S_{n}-S_{a}\right)}=0
\end{gathered}
$$

from which the following equilibrium prices obtain:

$$
P_{n}^{D}=\frac{2 \bar{\theta} S_{n}\left(S_{n}-S_{a}\right)}{4 S_{n}-S_{a}}, \quad P_{a}^{D}=\frac{\bar{\theta} S_{a}\left(S_{n}-S_{a}\right)}{4 S_{n}-S_{a}}
$$

In the first stage, firms choose quality specification $S_{n}^{D}$ and $S_{a}^{D}$ maximizing their profits, according to the following first order conditions ${ }^{1}$ :

$$
\begin{gathered}
\frac{\partial \Pi_{n}^{D}}{\partial S_{n}}=\frac{4 \bar{\theta}^{2}\left(4 S_{n}^{2}-3 S_{a} S_{n}+2 S_{a}^{2}\right) S_{n}}{\left(4 S_{n}-S_{a}\right)^{3}}-\gamma \cdot S_{n}=0 \\
\frac{\partial \Pi_{a}^{D}}{\partial S_{a}}=\frac{\bar{\theta}^{2}\left(4 S_{n}-7 S_{a}\right) S_{n}^{2}}{\left(4 S_{n}-S_{a}\right)^{3}}-\gamma \cdot S_{a}=0
\end{gathered}
$$

After substituting and re-arranging Equations (8) and (9), one obtains:

$$
8 S_{a}^{3}-12 S_{a}^{2} S_{n}+23 S_{n}^{2} S_{a}-4 S_{n}^{3}=0
$$

Set $\mu=S_{n} / S_{a}$, so that Equation (10) can be rewritten as:

$$
4 \mu^{3}-23 \mu^{2}+12 \mu-8=0
$$

The only solution (in the real numbers and greater than one) is $\mu^{*}=5.2512^{2}$. By substituting this value back into the first-order conditions, we obtain both equilibrium qualities:

$$
\begin{gathered}
S_{n}^{D=} \frac{4 \mu^{*} \bar{\theta}^{2}\left(4 \mu^{* 2}-3 \mu^{*}+2\right)}{\gamma\left(4 \mu^{*}-1\right)^{3}}=\frac{0.2533 \bar{\theta}^{2}}{\gamma}, S_{a}^{D}=\frac{\mu^{* 2} \bar{\theta}^{2}\left(4 \mu^{*}-7\right)}{\gamma\left(4 \mu^{*}-1\right)^{3}}=\frac{0.0482 \bar{\theta}^{2}}{\gamma} \\
S_{n}^{D}-S_{a}^{D}=\frac{0.2051 \bar{\theta}^{2}}{\gamma}
\end{gathered}
$$

Thus, the equilibrium duopoly qualities and the quality difference between incubative innovator and acquisi-

${ }^{1}$ Second-order conditions are satisfied throughout the calculations contained in this paper, although they are not displayed for the sake of brevity.

${ }^{2}$ Calculations and simulations were made with the software Mathematica. We cannot express straightforwardly the form of this root. 
tive innovator depend positively on the square of the upper bound of taste distribution, $\bar{\theta}$, and negatively on the R\&D cost parameter, $\gamma$, implying that the upper bound of taste parameter tends to increase both firms' innovation incentives, while the R\&D cost parameter tends to decrease both firms' innovation incentives.

Using the optimal qualities, we obtain the corresponding equilibrium prices, demand and profits:

$$
\begin{gathered}
P_{n}^{D}=\frac{8 \mu^{*} \bar{\theta}^{3}\left(\mu^{*}-1\right)\left(4 \mu^{* 2}-3 \mu^{*}+2\right)}{\gamma\left(4 \mu^{*}-1\right)^{4}}=\frac{0.1077 \bar{\theta}^{3}}{\gamma} \\
P_{a}^{D}=\frac{\mu^{* 2} \bar{\theta}^{3}\left(\mu^{*}-1\right)\left(4 \mu^{*}-7\right)}{\gamma\left(4 \mu^{*}-1\right)^{4}}=\frac{0.0103 \bar{\theta}^{3}}{\gamma} \\
d_{n}^{D}=\frac{2 \bar{\theta} \mu^{*}}{4 \mu^{*}-1}=0.5249 \bar{\theta} \\
d_{a}^{D}=\frac{\bar{\theta} \mu^{*}}{4 \mu^{*}-1}=0.2625 \bar{\theta} \\
\Pi_{n}^{D}=\frac{8 \mu^{* 3} \bar{\theta}^{4}\left(4 \mu^{*}-7\right)\left(4 \mu^{* 2}-3 \mu^{*}+2\right)}{\gamma\left(4 \mu^{*}-1\right)^{6}}=\frac{0.0244 \bar{\theta}^{4}}{\gamma} \\
\Pi_{a}^{D}=\frac{\mu^{* 3} \bar{\theta}^{4}\left(4 \mu^{*}-7\right)\left(4 \mu^{* 2}-3 \mu^{*}+2\right)}{2 \gamma\left(4 \mu^{*}-1\right)^{6}}=\frac{0.0015 \bar{\theta}^{4}}{\gamma}
\end{gathered}
$$

Since $\Pi_{n}^{D}>0$ and $\Pi_{a}^{D}>0$, both incubative innovator and acquisitive innovator will choose to enter the market in the first stage.

By comparing the equilibrium prices, demand and profits of both firms, we find out that the equilibrium price, demand and profit of incubative innovator are higher than the ones of acquisitive innovator, indicating highquality advantage continues to hold in this scenario.

\section{Triopoly Game after Shanzhai Phenomenon Emerged}

In this section, we introduce Shanzhai innovator into the model to depict the scenario after Shanzhai phenomenon emerged. Thus we analyze a model in which three firms compete [11], labeled $i=m, a$, $n$, indicating firms adopt Shanzhai mode, acquisitive innovation mode and incubative innovation mode respectively. As shown in the news report and academic researches, the quality of Shanzhai product is far lower than products developed by acquisitive innovator and incubative innovator. Hence, we assume that $S_{n}>S_{a}>S_{m} \geq 0$. Given consumer's utility function, we denote $\theta_{1}^{\prime}=P_{m} / S_{m}$ as the consumer indifferent between purchasing the product offered by Shanzhai innovator or not consuming at all. And $\theta_{2}^{\prime}=\left(P_{a}-P_{m}\right) /\left(S_{a}-S_{m}\right)$ represents the consumer indifferent between buying Shanzhai product or acquisitive innovator's product, and $\theta_{3}^{\prime}=\left(P_{n}-P_{a}\right) /\left(S_{n}-S_{a}\right)$ represents the consumer indifferent between consuming acquisitive innovator's product and incubative innovator's product. Denote the variables in this case with a superscript $T$. Thereby, the demand for three kinds of product are yield, with

$$
\begin{gathered}
d_{n}^{T}=\bar{\theta}-\theta_{3}^{\prime}=\bar{\theta}-\frac{P_{n}-P_{a}}{S_{n}-S_{a}} \\
d_{a}^{T}=\theta_{3}^{\prime}-\theta_{2}^{\prime}=\frac{P_{n}-P_{a}}{S_{n}-S_{a}}-\frac{P_{a}-P_{m}}{S_{a}-S_{m}} \\
d_{m}^{T}=\theta_{2}^{\prime}-\theta_{1}^{\prime}=\frac{P_{a}-P_{m}}{S_{a}-S_{m}}-\frac{P_{m}}{S_{m}}
\end{gathered}
$$

The R\&D cost for firms adopt incubative innovation mode and acquisitive innovation mode is the same as in 
the previous section, where $C\left(S_{n}\right)=\gamma \cdot S_{n}^{2} / 2$ and $C\left(S_{a}\right)=\gamma \cdot S_{a}^{2} / 2$ respectively. And the R\&D cost for firms adopted Shanzhai innovation mode is $C\left(S_{m}\right)=\gamma \cdot \beta \cdot S_{n}^{2} / 2, \beta \in(0,1)$, where $\beta$ is the efficiency parameter of imitative activities. Efficiency parameter $\beta$ depends on Shanzhai innovator's learning capability and the feasibility to imitate. Lower value of $\beta$ indicates higher learning capability and higher feasibility of imitation. Thus the profits are given by:

$$
\begin{gathered}
\pi_{n}^{T}=\left(\bar{\theta}-\frac{P_{n}-P_{a}}{S_{n}-S_{a}}\right) P_{n}-\frac{\gamma \cdot S_{n}^{2}}{2} \\
\pi_{a}^{T}=\left(\frac{P_{n}-P_{a}}{S_{n}-S_{a}}-\frac{P_{a}-P_{m}}{S_{a}-S_{m}}\right) P_{a}-\frac{\gamma \cdot S_{a}^{2}}{2} \\
\pi_{m}^{T}=\left(\frac{P_{a}-P_{m}}{S_{a}-S_{m}}-\frac{P_{m}}{S_{m}}\right) P_{m}-\frac{\gamma \cdot \beta \cdot S_{m}^{2}}{2}
\end{gathered}
$$

Similarly, firms engage in a two-stage game choosing, first, whether to enter the market and quality levels, then prices. Using the same methodology, we obtain the equilibrium prices as functions of quality levels:

$$
\begin{gathered}
P_{n}^{T}=\frac{\left(S_{n}-S_{a}\right)\left(4 S_{n} S_{a}-3 S_{a} S_{m}-S_{n} S_{m}\right) \bar{\theta}}{2 \tau} \\
P_{a}^{T}=\frac{S_{a}\left(S_{n}-S_{a}\right)\left(S_{a}-S_{m}\right) \bar{\theta}}{\tau} \\
P_{m}^{T}=\frac{S_{m}\left(S_{n}-S_{a}\right)\left(S_{a}-S_{m}\right) \bar{\theta}}{2 \tau}
\end{gathered}
$$

where $\tau \equiv 4 S_{a} S_{n}-S_{a}^{2}-2 S_{a} S_{m}-S_{m} S_{n}>0$. Thus we obtain the corresponding equilibrium demand:

$$
\begin{gathered}
d_{n}^{T}=\frac{\left(4 S_{n} S_{a}-3 S_{a} S_{m}-S_{n} S_{m}\right) \bar{\theta}}{2 \tau} \\
d_{a}^{T}=\frac{S_{a}\left(S_{n}-S_{m}\right) \bar{\theta}}{\tau} \\
d_{m}^{T}=\frac{S_{a}\left(S_{n}-S_{a}\right) \bar{\theta}}{2 \tau}
\end{gathered}
$$

Substituting these values into Equations (23)-(25) above, one can obtain the profit functions defined in terms of qualities only:

$$
\begin{gathered}
\pi_{n}^{T}=\frac{\left(S_{n}-S_{a}\right)\left(4 S_{n} S_{a}-3 S_{a} S_{m}-S_{n} S_{m}\right)^{2} \bar{\theta}^{2}}{4 \tau^{2}}-\frac{\gamma \cdot S_{n}^{2}}{2} \\
\pi_{a}^{T}=\frac{\left(S_{n}-S_{a}\right)\left(S_{a}-S_{m}\right)\left(S_{n}-S_{m}\right) S_{a}^{2} \bar{\theta}^{2}}{\tau^{2}}-\frac{\gamma \cdot S_{a}^{2}}{2} \\
\pi_{m}^{T}=\frac{S_{m} S_{a}\left(S_{a}-S_{m}\right)\left(S_{n}-S_{a}\right)^{2} \bar{\theta}^{2}}{4 \tau^{2}}-\frac{\gamma \cdot \beta \cdot S_{m}^{2}}{2}
\end{gathered}
$$

In the first stage, firms choose quality specification $S_{n}^{T}, S_{a}^{T}$ and $S_{m}^{T}$ maximizing their profits, according to the following first order conditions:

$$
\begin{aligned}
\frac{\partial \pi_{n}^{T}}{\partial S_{n}}= & \bar{\theta}^{2}\left[8 S_{a}^{5}\left(3 S_{m}-4 S_{n}\right)+6 S_{a} S_{m}^{2}\left(S_{m}-2 S_{n}\right) S_{n}^{2}+S_{m}^{3} S_{n}^{3}+S_{a}^{4}\left(48 S_{n}^{2}-21 S_{m}^{2}\right)\right. \\
& \left.+S_{a}^{2} S_{m} S_{n}\left(17 S_{m}^{2}-45 S_{m} S_{n}+48 S_{n}^{2}\right)+S_{a}^{3}\left(24 S_{m}^{3}-66 S_{m}^{2} S_{n}+72 S_{m} S_{n}^{2}-64 S_{n}^{3}\right)\right] / 4 \tau^{3}-\gamma \cdot S_{n}=0
\end{aligned}
$$




$$
\begin{gathered}
\frac{\partial \pi_{a}^{T}}{\partial S_{a}}=\frac{\bar{\theta}^{2}\left[S_{a}\left(S_{m}-S_{n}\right) S_{a}^{3}\left(5 S_{m}-7 S_{n}\right)+2 S_{m}^{2} S_{n}^{2}-3 S_{a} S_{m} S_{n}\left(S_{m}+S_{n}\right)+S_{a}^{2}\left(4 S_{m} S_{n}-2 S_{m}^{2}+4 S_{n}^{2}\right)\right]}{4 \tau^{3}}-\gamma \cdot S_{a}=0 \\
\frac{\partial \pi_{m}^{T}}{\partial S_{m}}=S_{a}^{2}\left(S_{a}-S_{n}\right)^{2}\left[S_{a}^{2}+7 S_{m} S_{n}-4 S_{a}\left(S_{m}+S_{n}\right)\right] \bar{\theta}^{2} / 4 \tau^{3}-\gamma \cdot \beta \cdot S_{m}=0
\end{gathered}
$$

Since the system (35)-(37) cannot be solved analytically, I resort to numerical calculations, letting $\bar{\theta}=1$, $\gamma=1, \beta$ vary in the interval $(0,1]$. The results of the simulation are displayed in Table 1 , where consumer surplus $(C S)$, producer surplus $(P S)$, and social welfare $(S W)$ are also compared ${ }^{3}$.

Thus we can summarize the equilibrium outcomes as follows:

1) The profit of Shanzhai innovator will be greater than that of acquisitive innovator and incubative innovator if imitation is feasible and the learning capability of Shanzhai innovator is sufficiently high ( $\beta$ small), but it will be smaller otherwise ( $\beta$ large).

2) Smaller efficiency parameter of imitative activities increases the quality level of Shanzhai innovator, while decrease the quality level of acquisitive innovator and incubative innovator. It implies that acquisitive innovator and incubative innovator tend to have lower innovation incentive when imitation is feasible and the learning capability of Shanzhai innovator is high. Smaller efficiency parameter also decreases the quality difference between products. The decrease of quality difference intensifies price competition and therefore decreases the price and profit of acquisitive innovator and incubative innovator.

3) Smaller efficiency parameter of imitative activities increases the market shares of Shanzhai innovator and acquisitive innovator, while decreases the market shares of incubative innovator.

4) Smaller efficiency parameter of imitative activities decreases producer surplus, and increases consumer surplus and social welfare.

\section{Comparing Equilibria}

In order to compare the equilibrium results before and after the emergence of Shanzhai phenomenon, we also resort to numerical calculations, setting $\bar{\theta}=1, \beta=0.5, \gamma$ vary in the interval $[1,50]$. The results of the simulation are displayed in Table 2 and Table 3.

By comparing the equilibrium results of the two games, we can obtain that:

1) The entry of Shanzhai innovator decreases the quality level of incubative innovator, while increases the quality level of acquisitive innovator. Noted that $S_{n}^{D}>S_{n}^{T}, S_{a}^{D}<S_{a}^{T}$, hence, we claim that the entry of Shanzhai innovator has opposite impact on the quality level of the other two rivals. Incubative innovator tends to have lower innovation incentive and acquisitive innovator tends to invest more on innovation if Shanzhai innovator enters the market.

2) The entry of Shanzhai innovator mildly decreases the price and the profit of both incubative innovator and acquisitive innovator. As shown above, $P_{a}^{D}>P_{a}^{T}, P_{n}^{D}>P_{n}^{T}, \Pi_{a}^{D}>\Pi_{a}^{T}, \Pi_{n}^{D}>\Pi_{n}^{T}$, and that's because the entry of Shanzhai innovator reduces the quality difference between products, which intensify the price competition among firms and leads to a decline in the profits of acquisitive innovator and incubative innovator .

3) The entry of Shanzhai innovator increases the market share of acquisitive innovator, but decreases the market share of incubative innovator $\left(d_{a}^{D}<d_{a}^{T}, d_{n}^{D}>d_{n}^{T}\right)$. And significant increase in the market share of the whole industry is seen if Shanzhai innovator chooses to enter the market $\left(d^{D}<d^{T}\right)$. That's because Shanzhai products fill the gap left by acquisitive innovator and incubative innovator by capturing the low-end of the market.

4) The entry of Shanzhai innovator decreases producer surplus and social welfare, while increases consumer surplus $\left(\Pi^{D}>\Pi^{T}, S W^{D}>S W^{T}, C S^{D}<C S^{T}\right)$, implying that though consumers are better off when Shanzhai

${ }^{3}$ Consumer Surplus is the consumers' net utilityand it is given by the following formula:

$$
C S^{T}=\int_{\theta_{1}^{\prime}}^{\theta_{2}^{\prime}}\left(\theta S_{m}-P_{m}\right) \mathrm{d} \theta+\int_{\theta_{2}^{\prime}}^{\theta_{3}^{\prime}}\left(\theta S_{a}-P_{a}\right) \mathrm{d} \theta+\int_{\theta_{3}^{\prime}}^{\bar{\theta}}\left(\theta S_{n}-P_{n}\right) \mathrm{d} \theta .
$$

Producer Surplus is the sum of the net profits of all the producers in the industry. In this case, producer surplus is given by $P S^{T}=\Pi_{n}^{T}+\Pi_{a}^{T}+\Pi_{m}^{T}$. Social Welfare, defined as the sum of the producer and the consumer surpluses, is given by $S W^{T}=P S^{T}+C S^{T}$. 
Table 1. Equilibrium outcomes of the model after Shanzhai phenomenon emerged.

\begin{tabular}{cccccc}
\hline$\beta$ & 1 & 0.5 & 0.25 & 0.1 & 0.05 \\
\hline$S_{n}^{T}$ & 0.25267 & 0.25232 & 0.25199 & 0.25169 & 0.25156 \\
$S_{a}^{T}$ & 0.04972 & 0.05144 & 0.05408 & 0.05766 & 0.05958 \\
$S_{m}^{T}$ & 0.00953 & 0.01580 & 0.02283 & 0.03049 & 0.03418 \\
$P_{n}^{T}$ & 0.10604 & 0.10466 & 0.10281 & 0.10051 & 0.09931 \\
$P_{a}^{T}$ & 0.00913 & 0.00844 & 0.00771 & 0.00698 & 0.00665 \\
$P_{m}^{T}$ & 0.00088 & 0.00130 & 0.00163 & 0.00185 & 0.00191 \\
$d_{n}^{T}$ & 0.52249 & 0.52100 & 0.51949 & 0.51799 & 0.51731 \\
$d_{a}^{T}$ & 0.27213 & 0.27867 & 0.28579 & 0.29297 & 0.29616 \\
$d_{m}^{T}$ & 0.11358 & 0.11833 & 0.12341 & 0.12849 & 0.13077 \\
$d^{T}$ & 0.90820 & 0.91800 & 0.92869 & 0.93945 & 0.94424 \\
$\Pi_{n}^{T}$ & 0.02348 & 0.02269 & 0.02166 & 0.02039 & 0.01973 \\
$\Pi_{a}^{T}$ & 0.00125 & 0.00103 & 0.00074 & 0.00038 & 0.00019 \\
$\Pi_{m}^{T}$ & 0.00005 & 0.00009 & 0.00014 & 0.00019 & 0.00022 \\
$P S^{T}$ & 0.02479 & 0.02381 & 0.02254 & 0.02096 & 0.02015 \\
$C S^{T}$ & 0.04432 & 0.04532 & 0.04668 & 0.04842 & 0.04933 \\
$S W^{T}$ & 0.06911 & 0.06913 & 0.06922 & 0.06938 & 0.06948 \\
\hline
\end{tabular}

${ }^{\text {a }} d$ indicates market share of the whole industry.

Table 2. Equilibrium outcomes of the duopoly game.

\begin{tabular}{ccccccc}
\hline$\gamma$ & 1 & 2 & 5 & 10 & 20 & 50 \\
\hline$S_{n}^{D}$ & 0.253311 & 0.126655 & 0.050662 & 0.025331 & 0.012666 & 0.005066 \\
$S_{a}^{D}$ & 0.048238 & 0.024119 & 0.009648 & 0.004824 & 0.002412 & 0.000965 \\
$P_{n}^{D}$ & 0.107662 & 0.053831 & 0.021532 & 0.010766 & 0.005383 & 0.002153 \\
$P_{a}^{D}$ & 0.010251 & 0.005126 & 0.00205 & 0.001025 & 0.000513 & 0.000205 \\
$d_{n}^{D}$ & 0.524994 & 0.524994 & 0.524994 & 0.524994 & 0.524994 & 0.524994 \\
$d_{a}^{D}$ & 0.262497 & 0.262497 & 0.262497 & 0.262497 & 0.262497 & 0.262497 \\
$d^{D}$ & 0.787491 & 0.787491 & 0.787491 & 0.787491 & 0.787491 & 0.787491 \\
$\Pi_{n}^{D}$ & 0.024439 & 0.012219 & 0.004888 & 0.002444 & 0.001222 & 0.000489 \\
$\Pi_{a}^{D}$ & 0.001527 & 0.000764 & 0.000305 & 0.000153 & 0.000076 & 0.000031 \\
$\Pi^{D}$ & 0.025966 & 0.012983 & 0.005193 & 0.002597 & 0.001298 & 0.000519 \\
$C S^{D}$ & 0.043218 & 0.021609 & 0.008644 & 0.004322 & 0.002161 & 0.000864 \\
$S W^{D}$ & 0.069184 & 0.034592 & 0.013837 & 0.006918 & 0.003459 & 0.001384 \\
\hline
\end{tabular}

innovator enters the market, producer surpluses decrease and more than offset the positive effects over consumers. Thus, Shanzhai innovator is not welcomed by its rivals and the social planer.

\section{Concluding Remarks}

In this paper, we focus on the impact of the emergence of Shanzhai phenomenon, and analyze and compare the equilibrium result of the competition among different innovation modes before and after the entry of Shanzhai 
Table 3. Equilibrium outcomes of the triopoly game.

\begin{tabular}{ccccccc}
\hline$\gamma$ & 1 & 2 & 5 & 10 & 20 & 50 \\
\hline$S_{n}^{T}$ & 0.252316 & 0.126158 & 0.050463 & 0.025232 & 0.012616 & 0.005046 \\
$S_{a}^{T}$ & 0.051444 & 0.025722 & 0.010289 & 0.005144 & 0.002572 & 0.001029 \\
$S_{m}^{T}$ & 0.015795 & 0.007898 & 0.003159 & 0.001580 & 0.000790 & 0.000316 \\
$P_{n}^{T}$ & 0.104655 & 0.052327 & 0.020931 & 0.010466 & 0.005233 & 0.002093 \\
$P_{a}^{T}$ & 0.008437 & 0.004218 & 0.001687 & 0.000844 & 0.000422 & 0.000169 \\
$P_{m}^{T}$ & 0.001295 & 0.000648 & 0.000259 & 0.000130 & 0.000065 & 0.000026 \\
$d_{n}^{T}$ & 0.521 & 0.521 & 0.521 & 0.521 & 0.521 & 0.521 \\
$d_{a}^{T}$ & 0.278667 & 0.278667 & 0.278667 & 0.278667 & 0.278667 & 0.278667 \\
$d_{m}^{T}$ & 0.118333 & 0.118333 & 0.118333 & 0.118333 & 0.118333 & 0.118333 \\
$d^{T}$ & 0.918 & 0.918 & 0.918 & 0.918 & 0.918 & 0.918 \\
$\Pi_{n}^{T}$ & 0.022693 & 0.011347 & 0.004539 & 0.002269 & 0.001135 & 0.000454 \\
$\Pi_{a}^{T}$ & 0.001028 & 0.000514 & 0.000206 & 0.000103 & 0.000051 & 0.000021 \\
$\Pi_{m}^{T}$ & 0.000091 & 0.000045 & 0.000018 & 0.000009 & 0.000005 & 0.000002 \\
$\Pi^{T}$ & 0.023812 & 0.011906 & 0.004762 & 0.002381 & 0.001191 & 0.000476 \\
$C S^{T}$ & 0.045316 & 0.022658 & 0.009063 & 0.004532 & 0.002266 & 0.000906 \\
$S W^{T}$ & 0.069128 & 0.034564 & 0.013826 & 0.006913 & 0.003456 & 0.001383 \\
\hline
\end{tabular}

innovator. We assume that the R\&D costs of the three innovation modes are asymmetry and the products are differentiated. Based on vertical product differentiation model, we analyze the duopoly game between incubative innovation and acquisitive innovation before Shanzhai phenomenon emerged, and analyze the triopoly game among incubative innovation, acquisitive innovation and Shanzhai mode after Shanzhai phenomenon emerged. We observe that, in the duopoly game, incubative innovator reaps larger profits than acquisitive innovator, indicating that high-quality advantage holds before the entry of Shanzhai innovator. In the triopoly game, the highquality advantage needs not to hold when the efficiency parameter of imitative activities is sufficiently small. By comparing the equilibrium result of the two games, we find that the entry of Shanzhai innovator decreases the quality level of incubative innovator while increases the quality level of acquisitive innovator. Prices and profits of both acquisitive innovator and incubative innovator decrease when Shanzhai innovator joins the competition. Shanzhai innovator's entry also decreases producer surplus and social welfare, but increases consumer surplus, implying that more efforts are needed for the government to guide and discipline imitative activities of Shanzhai innovator in a way that creates win-win-win situation for innovators and consumers. As to Shanzhai innovator, it should be noted that, high-quality advantage could not be reversed unless imitation is feasible and Shanzhai innovator possesses superior learning capability. Therefore, on one hand, Shanzhai innovator should develop its learning capability, and on the other hand, it should make active transformation in case imitation activities become impossible.

\section{References}

[1] Teece, D.J. (2007) Explicating Dynamic Capabilities: The Nature and Microfoundations of (Sustainable) Enterprise Performance. Strategic Management Journal, 28, 1319-1350. http://dx.doi.org/10.1002/smj.640

[2] Hull, C.E.I.K. and Covin, J.G. (2010) Learning Capability, Technological Parity, and Innovation Mode Use. Journal of Product Innovation Management, 27, 97-114. http://dx.doi.org/10.1111/j.1540-5885.2009.00702.x

[3] Zahra, S.A. and Covin, J.G. (1994) The Financial Implications of Fit between Competitive Strategy and Innovation Types and Sources. The Journal of High Technology Management Research, 5, 183-211. http://dx.doi.org/10.1016/1047-8310(94)90002-7 
[4] Luo, Y., Sun, J. and Wang, S.L. (2011) Emerging Economy Copycats: Capability, Environment, and Strategy. The Academy of Management Perspectives, 25, 37-56. http://dx.doi.org/10.5465/AMP.2011.61020801

[5] Li, Y., Zhang, C., Liu, Y. and Li, M. (2010) Organizational Learning, Internal Control Mechanisms, and Indigenous Innovation: The Evidence from China. IEEE Transactions on Engineering Management, 57, 63-77. http://dx.doi.org/10.1109/TEM.2009.2028321

[6] Hung, S. and Tang, R. (2008) Factors Affecting the Choice of Technology Acquisition Mode: An Empirical Analysis of the Electronic Firms of Japan, Korea and Taiwan. Technovation, 28, 551-563. http://dx.doi.org/10.1016/j.technovation.2007.10.005

[7] Kao, H. and Lee, J. (2010) An Investigation into the Key Elements of the Chinese Shanzhai Model: Alternative Path to Growth, Cross-Specialization Partnership, and Opportunistic Niche Infiltration. Proceedings of Technology Management for Global Economic Growth (PICMET), 1-13.

[8] Peppal, L. (1997) Imitative Competition and Product Innovation in a Duopoly Model. Economica, 64, $265-279$. http://dx.doi.org/10.1111/1468-0335.00077

[9] Lehmann-Grube, U. (1997) Strategic Choice of Quality When Quality Is Costly: The Persistence of the High-Quality Advantage. The Rand Journal of Economics, 28, 372-385. http://dx.doi.org/10.2307/2555811

[10] Motta, M. (1993) Endogenous Quality Choice: Price vs. Quantity Competition. The Journal of Industrial Economics, 113-131. http://dx.doi.org/10.2307/2950431

[11] Scarpa, C. (1998) Minimum Quality Standards with More than Two Firms. International Journal of Industrial Organization, 16, 665-676. http://dx.doi.org/10.1016/S0167-7187(96)01051-X 